\title{
Effect of glucosamine on intraocular pressure: a randomized clinical trial
}

\begin{abstract}
Purpose The purpose of the study was to investigate ocular hypertensive effect of exogenous glucosamine in comparison with placebo in patients with osteoarthritis.

Patients and methods In this double-masked randomized clinical trial, 88 patients with osteoarthritis were included. Forty-four patients were randomized into either glucosamine sulfate or the placebo group. Comprehensive ophthalmologic exam including intraocular pressure (IOP) at baseline, month 1, and 3 was performed. Ocular response analyzer parameters were also checked at baseline and month 3 . Results The mean IOP at the time of presentation was $12.4 \pm 2.7 \mathrm{~mm} \mathrm{Hg}$ in glucosamine and $13 \pm 2.8 \mathrm{~mm} \mathrm{Hg}$ in the placebo group $(P=0.329)$. At month 1 the corresponding values were $12.6 \pm 2.4$ and $12.9 \pm 2.4 \mathrm{~mm} \mathrm{Hg}(P=0.868)$, and at 3 months follow-up were $13.5 \pm 2.3$ and $13 \pm 2.7 \mathrm{~mm} \mathrm{Hg}$ $(P=0.002)$, respectively. About $34.1 \%$ in treatment and $12.5 \%$ in the placebo group had clinically significant (defined as $\geq 2 \mathrm{~mm} \mathrm{Hg}$ ) rise in IOP at final follow-up $(P=0.023)$. Mean age in those with significant rise in IOP was 66 vs 57.7 years in patients with $<2 \mathrm{~mm} \mathrm{Hg}(P=0.034)$. The ORA parameters remained unchanged in both the groups during the course of study.

Conclusion Glucosamine supplement therapy causes statistically significant rise of IOP, which is more pronounced in elderly patients. Clinical implication of this finding needs further evaluation.
\end{abstract}

Eye (2017) 31, 389-394; doi:10.1038/eye.2016.221; published online 21 October 2016

\section{Introduction}

Over the last decade, nutritional supplements have emerged as a new treatment for
H Esfandiari ${ }^{1}$, M Pakravan², Z Zakeri ${ }^{3}$, S Ziaie $^{4}$, P Pakravan ${ }^{5}$ and $V$ Ownagh ${ }^{1}$

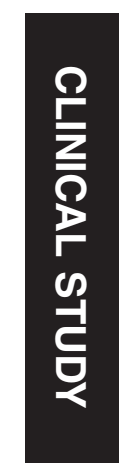

management of osteoarthritis (OA). ${ }^{1}$ These medications generally fall into two categories as either structure- or symptoms-modifying agents. ${ }^{2}$ Glucosamine is the most common prescribed symptomatic slow-acting drugs for $\mathrm{OA}$ and has proven to interfere with disease progression. ${ }^{3,4}$ It is an amino monosaccharide and essential constituent of chondroitin and keratin sulfate, principal glycosaminoglycans (GAG) in the cartilage. ${ }^{5}$ Although glucosamine is the main component of the cartilage, it is also abundant in corneal stroma and contribute morphological and functional characteristics to trabecular meshwork. ${ }^{6,7}$ Glucosamine is frequently prescribed for patients with $\mathrm{OA}$, and radiographic studies have shown its efficacy in slowing joint space width loss. ${ }^{8,9}$ In most countries oral glucosamine supplement is available as over the counter medication. Till now, the most important advantage of this medication is its safety profile. ${ }^{10}$

Despite a large number of patients using this medication, evidences have emerged that refused effectiveness in OA-related pain and functional score, ${ }^{11}$ and also one small study raised the possible association of rise of intraocular pressure (IOP) following glucosamine supplement usage. ${ }^{12}$

In this randomized clinical trial, we decided to explore the possible role of glucosamine supplement in rise of IOP in patients with OA.

\section{Subjects and methods}

This study was a double-masked blockrandomized clinical trial to evaluate the effect of glucosamine supplement on IOP and corneal biomechanical characteristics. Eighty-eight patients with OA were recruited from rheumatology clinic from July 2014 to March 2015. The study was approved by the ethics committee (an equivalent to Institutional Review
${ }^{1}$ Department of Ophthalmology, Ophthalmic Research Center, Labbafinejad Medical Center, Shahid Beheshti University of Medical Sciences, Tehran, Iran

2Ophthalmic Epidemiology Research Center, Shahid Beheshti University of Medical Sciences, Tehran, Iran

${ }^{3}$ Department of Internal Medicine, Labbafinejad Medical Center, Shahid Beheshti University of Medical Sciences, Tehran, Iran

${ }^{4}$ Department of Clinical Pharmacy, School of Pharmacy, Shahid Beheshti University of Medical Sciences, Tehran, Iran

${ }^{5}$ Orange Coast Colleges, Costa Mesa, CA, USA

Correspondence: H Esfandiari, Department of Ophthalmology, Ophthalmic Research Center, Labbafinejad Medical Center, Shahid Beheshti University of Medical Sciences, Pasdaran Ave. Boostan 9 St, Tehran 1666694516, Iran Tel: +98 2122562128 ; Fax: +98 2122590607. Email: hmdesfandiary@ gmail.com

Received: 26 April 2016 Accepted in revised form: 8 September 2016 Published online: 21 October 2016 
Boards) of the Ophthalmic Research Center of Shahid Beheshti University of Medical Science and registered at http:/ / www.clinicaltrials.gov (NCT02306083) according to the standards set by the International Committee of Medical Journal editors and the World Health Organization. After receiving adequate explanation about the natural course of the disease and our study therapeutic options, written informed consent was obtained from all patients prior to enrollment.

Inclusion criteria were all patients with OA referred to rheumatology clinic and written consent form. Exclusion criteria were any ophthalmological disease with possible effect on biomechanics of the cornea including history of any ocular surgery, corneal scar, and dystrophies.

Moreover, patients with poor performance on study tests were excluded.

Randomization was performed using automated, computerized permuted block-randomization lists with a random varying block size of 2 and 4 .

Both rheumatologist who had visited the patient at the first time, and ophthalmologist who performed ophthalmic exam, were masked to randomization. Patients were supplied with medication by third nonmasked investigator. Masking was reserved till all data were extracted from patients' files.

Patients were randomly assigned to group A or the treatment group and group B or the placebo group, and treated either with glucosamine sulfate or placebo, $750 \mathrm{mg}$ three times a day for a 3 months duration.

Both glucosamine sulfate and placebo tablets (manufactured by Avicenna Laboratories Inc., Tehran, Iran) had exactly the same appearance and were bottled in the same containers.

A clinical pharmacist visited the patients monthly and delivered the supplements to them to enhance the patients' adherence. In the first visit, all demographic data, comorbidities, and drug history were collected and a case report form (CRF) was filled for each participant. During all the next visits safety of supplements were evaluated by the clinical pharmacist and any drug side effects expressed by the patients were recorded.

All patients underwent comprehensive ophthalmic examinations at baseline, and months 1 and 3 . Visual field testing and peripapillary retinal nerve fiber layer thickness (PRNFLT) measurement were requested if glaucoma was suspected.

The 24-2 SITA standard visual field testing was performed using the Humphrey Visual Field Analyzer (HFA; model 750; Carl Zeiss Meditec, Inc., Dublin, CA, USA), and PRNFLT measured by optical coherence tomography (Cirrus HDOCT; Carl Zeiss Meditec). IOP measurements, as the main variable, were obtained with Goldmann applanation tonometer (GAT; Haag-Streit, Konig, Switzerland) by the ophthalmologist masked to the randomization. Average of two measurements per eye was considered for the analysis, and if two measurements differed more than $2 \mathrm{~mm} \mathrm{Hg}$, the third one was obtained and average of closest measurements was considered for statistical analysis. All measurements were taken at the same sitting with 5 min interval.

Corneal biomechanics was evaluated using ocular response analyzer (ORA; Reichert Inc., Depew, NY, USA) at baseline and month 3 . Four readings with acceptable wave forms were acquired and the mean of best three readings was included for analysis. ${ }^{13}$

Sample size was calculated to be 44 in each group to have $95 \%$ power to detect $2 \mathrm{~mm} \mathrm{Hg}$ difference between the two groups when the standard deviation of IOP change in both the groups assumed to be $2.5 \mathrm{~mm} \mathrm{Hg}$. In this calculation type I error was 0.05 and test considered to be two sided. To present data we used mean, standard deviation, median, and range. To compare the variables in the baseline, $t$-test and $\chi^{2}$-square test were used for subject's variable. To assess the equivalence of variance between the two groups we used Levene's test. For eyerelated variables these comparisons were performed by linear mixed model (LMM). Also, generalized LMM was used to compare the significant change ( $\geq 2 \mathrm{~mm} \mathrm{Hg}$ ) in IOP. All statistical analyses were performed by SPSS (IBM Corp. Released 2013. IBM SPSS Statistics for Windows, Version 22.0. Armonk, NY, USA: IBM Corp.). P-value $<0.05$ was considered statistically significant.

\section{Results}

A total of 88 patients, 67 female and 21 male, with a mean age of 57.7 years (range, 36-83) were enrolled from 185 candidates who were requested to participate in the study (47\%, Figure 1, Table 1). Participants were randomized equally into treatment and placebo groups, and were followed for 3 months.

The mean IOP at the time of presentation was $12.4 \pm 2.7 \mathrm{~mm} \mathrm{Hg}$ in glucosamine and $13 \pm 2.8 \mathrm{~mm} \mathrm{Hg}$ in the placebo group $(P=0.329)$. At month 1 the corresponding values were $12.6 \pm 2.4$ and $12.9 \pm 2.4 \mathrm{~mm} \mathrm{Hg},(P=0.868)$ and at 3 months follow-up were $13.5 \pm 2.3$ and $13 \pm 2.7 \mathrm{~mm} \mathrm{Hg}(P=0.002)$, respectively (Table 2, Figure 2). About $34.1 \%$ in the treatment and $12.5 \%$ in the placebo group had clinically significant (defined as $\geq 2 \mathrm{~mm} \mathrm{Hg}$ ) rise in IOP at final follow-up $(P=0.023)$.

The respective values for baseline ORA Goldmanncorrelated IOP (IOPg), cornea-compensated IOP (IOPcc), corneal hysteresis, and resistant factor (CRF) were $14.5 \pm 2.7,15.2 \pm 2.7,9.6 \pm 1.4$, and $9.5 \pm 1.3$ in the treatment group. The corresponding values in the placebo group were $15.3 \pm 3.7,15.5 \pm 3.6,10.5 \pm 1.7$, and $10.1 \pm 2.2$, 


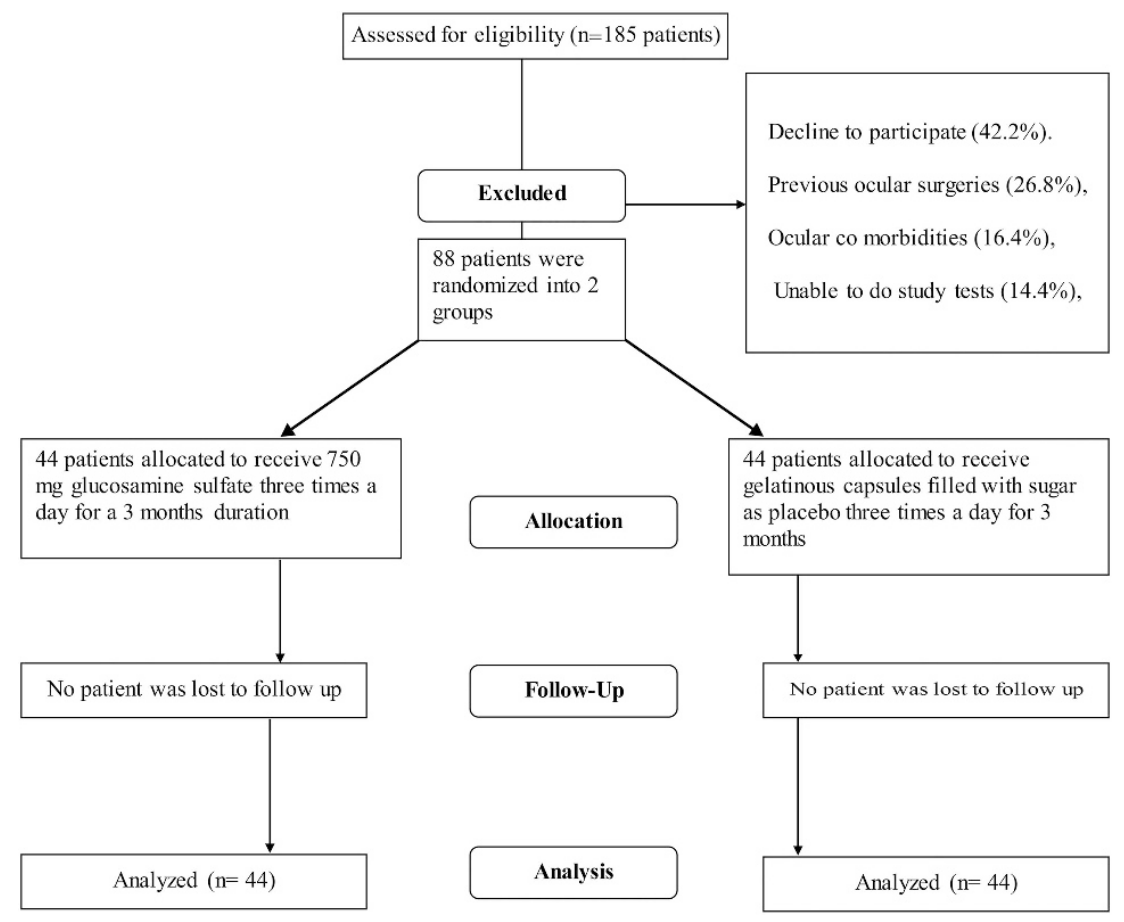

Figure 1 Flow chart showing progression of subjects through trial.

respectively (Table 1 , respective $P$-values: $0.276,0.682$, 0.008 , and 0.075).

At month 3 ORA parameters changed insignificantly to $14.8 \pm 2.7,15.3 \pm 2.5,9.5 \pm 1.3$, and $9.4 \pm 1.2$ in the treatment group, respectively (Table 2). Three-month ORA values in the placebo group also changed insignificantly to $14.3 \pm 4.2,14.5 \pm 4.3,10.4 \pm 1.7$, and $9.9 \pm 1.7$, respectively (Table 2 , all $P$-values $>0.05$ ). Levene's test showed that there was no statistically significant difference between the two groups regarding the variance $(P=0.340)$.

In regard to possible risk factors of diabetes mellitus, cardiovascular disease, gender, and age, only age was significantly associated with risk of ocular hypertension in the treatment group (respective $P$-values $=0.228,0.438$, 0.103 , and 0.034 ). Mean age in those with significant rise in IOP was 66 vs 57.7 years in patients with $<2 \mathrm{~mm} \mathrm{Hg}$ increased in IOP.

Among those using glucosamine supplement, only one patient complained of GI upset during the first month of trial.

\section{Discussion}

The results of this study show that while glucosamine causes statistically significant increase in IOP in patients with OA, corneal biomechanics remain unchanged within 3 months of glucosamine supplement therapy. Although mean rise of IOP was statistically significant in the glucosamine group, more than $2 \mathrm{~mm} \mathrm{Hg}$ rise in IOP was also more in the treatment group (34\% of those receiving treatment $v$ s $12.5 \%$ of patients on placebo).

The dietary supplement, glucosamine, has been advocated as primarily safe and effective treatment for management of OA. ${ }^{14}$ Meta analysis of early studies on effectiveness of glucosamine suggested potential advantages, but also questioned the scientific quality of the studies. ${ }^{14}$ A recent Cochrane review on glucosamine failed to show any improvement in pain and function of OA-related symptoms. ${ }^{11}$ The main advantage of glucosamine use has been considered its safety profile, both in short- and long-term studies. ${ }^{15}$ The proposed means of action is restoration of extracellular matrix of cartilage and/or halting additional cartilage degradation. ${ }^{16}$ Cornea and trabecular meshwork are also enriched with GAG, and it is no surprise if any change in the content of GAG affects IOP and its measurement. ${ }^{6}$

GAG have an important role in the morphology and function of the trabecular meshwork, and are advocated as a key physiological element of TM resistance. They constitute the ground substance for outer most part of the TM. ${ }^{17,18}$ Long and flexible chains of GAG interact with each other to form a system of entangled chains of polyanionic macromolecules, hence, act like gel, and are an important contributor to outflow resistance. ${ }^{7}$ 
Table 1 Patients' baseline characteristics and ORA parameters

\begin{tabular}{|c|c|c|c|}
\hline & Treatment & Placebo & P-value \\
\hline \multicolumn{4}{|l|}{ Age } \\
\hline Mean \pm SD & $60.6 \pm 11$ & $58.8 \pm 11.5$ & $0.73^{\mathrm{a}}$ \\
\hline \multicolumn{4}{|l|}{ Sex } \\
\hline Female & $33(75.0 \%)$ & $34(77.3 \%)$ & $0.803^{\mathrm{b}}$ \\
\hline \multicolumn{4}{|l|}{ Diabetes mellitus } \\
\hline No & $37(84.1 \%)$ & $40(90.9 \%)$ & $0.334^{\mathrm{b}}$ \\
\hline Yes & 7 (15.9\%) & $4(9.1 \%)$ & \\
\hline \multicolumn{4}{|l|}{ Hypertension } \\
\hline No & $33(75.0 \%)$ & $36(81.8 \%)$ & $0.437^{\mathrm{b}}$ \\
\hline Yes & $11(25.0 \%)$ & $8(18.2 \%)$ & \\
\hline \multicolumn{4}{|l|}{ Baseline IOP } \\
\hline Mean \pm SD & $12.4 \pm 2.7$ & $13 \pm 2.8$ & $0.329^{c}$ \\
\hline Median (range) & $12(8-19)$ & $12(10-20)$ & \\
\hline \multicolumn{4}{|l|}{ Baseline IOPcc } \\
\hline Mean \pm SD & $15.2 \pm 2.7$ & $15.5 \pm 3.6$ & $0.682^{c}$ \\
\hline \multicolumn{4}{|l|}{ Baseline IOPg } \\
\hline Mean $\pm S D$ & $14.5 \pm 2.7$ & $15.3 \pm 3.7$ & $0.276^{\mathrm{c}}$ \\
\hline \multicolumn{4}{|l|}{ Baseline CRF } \\
\hline Mean \pm SD & $9.5 \pm 1.3$ & $10.1 \pm 2.2$ & $0.075^{c}$ \\
\hline \multicolumn{4}{|l|}{ Baseline $\mathrm{CH}$} \\
\hline Mean \pm SD & $9.6 \pm 1.4$ & $10.5 \pm 1.7$ & $0.008^{c}$ \\
\hline
\end{tabular}

Abbreviations: $\mathrm{CH}$, corneal hysteresis; $\mathrm{CRF}$, corneal resistance factor; IOPcc, intraocular pressure corneal compensated; IOPg, intraocular pressure Goldmann correlated. ${ }^{\mathrm{a}} \mathrm{On}$ the basis of $t$-test. ${ }^{\mathrm{b}} \mathrm{On}$ the basis of $x^{2}$-test. ${ }^{\mathrm{C}} \mathrm{On}$ the basis of linear mixed model.

Binninger et $a l^{19}$ showed that exogenous GAG stimulate cultured human trabecular meshwork cells to produce more GAG as positive feedback mechanism.

Steroid-related trabecular meshwork changes and consequent ocular hypertension is a well-known disorder. Accumulation of GAG in the ground substance of the trabecular meshwork outflow pathways and its consequent constriction of the trabecular spaces, and increased outflow resistance has been proposed to explain rise of IOP following even short course of steroid therapy. ${ }^{20}$

In a small retrospective study, Murphy et al ${ }^{12}$ noted that oral glucosamine supplement was associated with significant but reversible rise in IOP. They observed poorly controlled glaucoma patients had their condition under control once glucosamine was discontinued, and also IOP increased in those patients with glaucoma who received glucosamine supplement for their OA condition. There were several weak points in their study that were also acknowledged by the authors, including variability in glucosamine dosage and length of treatment, compliance, and brand variability.

As GAG are also abundant in corneal stroma, ${ }^{21}$ any change in its concentration could lead to IOP measurements error as GAT is influenced by biomechanics of the cornea.

In the present study, biomechanics of the cornea remained unchanged within 3 months of treatment.

There is no study concerning pharmacokinetics of glucosamine in trabecular meshwork. Considering glucosamine access to target site in $\mathrm{OA}$, it has been shown that its concentration in synovial fluid following oral administration is 500 -fold less than the level expected to have therapeutic effect on joint space narrowing. ${ }^{22}$

On the basis of our results, it is possible that oral supplement could reach pathologic level at TM at least within 3 months of treatment. Another theory to explain this observation is that high concentration of GAG in aqueous draws more water into the anterior chamber as a result of osmotic effect, thus induces swelling and compromising of pore sizes, leading to high IOP. ${ }^{7}$

We did not find diabetes mellitus, cardiovascular disease, and gender as risk factors for glucosamineinduced hypertension; but age was significantly associated with rise of IOP in the treatment group. Several histological studies demonstrated age-related structural and functional changes including loss of cellularity, collagen and extracellular matrix accumulation, thickening and fusion of trabecular sheats. ${ }^{23-26}$ Age is also a risk factor for steroid-induced ocular hypertension. ${ }^{20}$ Accumulation of extracellular matrix and thickening of the basement membrane could be aggravated by exogenous use of GAG and compromise the reduced function of trabecular meshwork.

The number of glaucomatous patients or those with family history of glaucoma was not large enough to permit any statistical comparison, so we cannot comment on this subject; but considering compromised trabecular meshwork in these patients, it's a wise practice that ophthalmologists directly ask patients about its usage and carry out medication discontinuation trial in uncontrolled cases.

Although the strength of this study is its doublemasked randomized nature, it is limited by its relatively short follow-up period. Considering the significant change in IOP within short term of the previous study, ${ }^{12}$ we scheduled to follow the patients for 3 months. It is a possibility that GAG concentration in TM might depend on long-term dosing of treatment and longer duration of treatment leads to more pronounced rise of IOP.

One should interpret the outcome of our study with caution; although the rise of IOP in the study 
Table 2 IOP values at first and third month, and ORA parameters after 3 months

\begin{tabular}{|c|c|c|c|c|c|c|c|}
\hline & & \multirow[t]{2}{*}{ Treatment } & \multirow[t]{2}{*}{ Placebo } & \multicolumn{2}{|c|}{$95 \% C I$} & \multirow[t]{2}{*}{ P-value } & \multirow[t]{2}{*}{ P-value ${ }^{b}$} \\
\hline & & & & Lower & Upper & & \\
\hline \multicolumn{8}{|l|}{ Month 1 IOP } \\
\hline Value & Mean \pm SD & $12.6 \pm 2.4$ & $12.9 \pm 2.4$ & -1.1 & 0.4 & 0.868 & 0.783 \\
\hline Significant change & $n(\%)$ & $14(16.7 \%)$ & $9(11.3 \%)$ & $-5.3 \%$ & $16.2 \%$ & & \\
\hline \multicolumn{8}{|l|}{ Month 3 IOP } \\
\hline Value & Mean \pm SD & $13.5 \pm 2.3$ & $13 \pm 2.7$ & -0.3 & 1.3 & 0.002 & 0.005 \\
\hline Significant change & $n(\%)$ & $28(34.1 \%)$ & $10(12.5 \%)$ & $8.9 \%$ & $34.4 \%$ & 0.023 & 0.038 \\
\hline \multicolumn{8}{|l|}{ Month 3 IOPcC } \\
\hline Value & Mean \pm SD & $15.3 \pm 2.5$ & $14.5 \pm 4.3$ & -0.2 & 2.0 & 0.026 & 0.127 \\
\hline \multicolumn{8}{|l|}{ Month $3 \mathrm{IOPg}$} \\
\hline Value & Mean \pm SD & $14.8 \pm 2.7$ & $14.3 \pm 4.2$ & -0.5 & 1.6 & 0.035 & 0.196 \\
\hline \multicolumn{8}{|l|}{ Month 3 CRF } \\
\hline Value & Mean \pm SD & $9.4 \pm 1.2$ & $9.9 \pm 1.7$ & -1.0 & 0.0 & 0.471 & 0.025 \\
\hline \multicolumn{8}{|l|}{ Month $3 \mathrm{CH}$} \\
\hline Value & Mean \pm SD & $9.5 \pm 1.3$ & $10.4 \pm 1.7$ & -1.3 & -0.4 & 0.487 & 0.486 \\
\hline
\end{tabular}

Abbreviation: CI, confidence interval. adjusted for the baseline value, based on linear mixed model. ${ }^{\mathrm{b}}$ Adjusted for the baseline value, age, sex, DM and HTN status, based on linear mixed model.

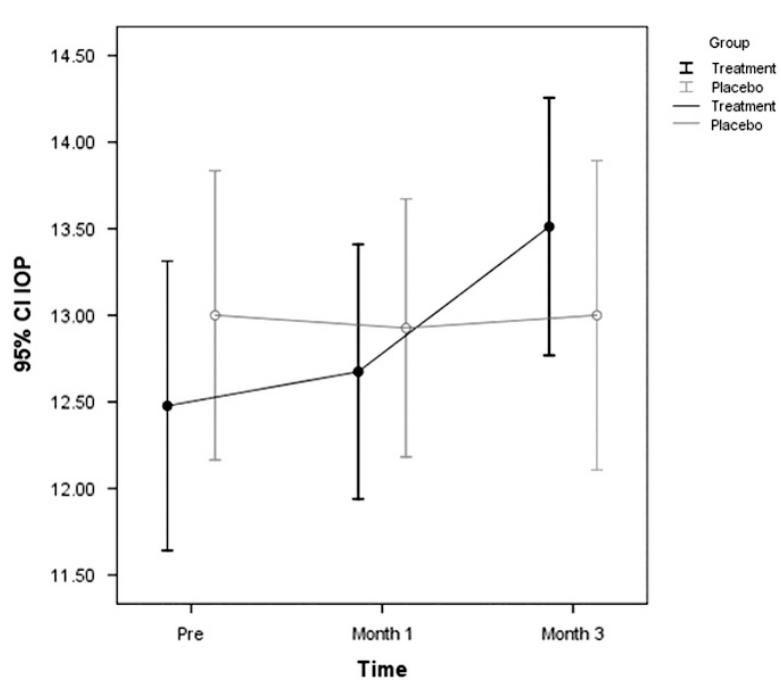

Figure 2 IOP changes in each group within the course of study.

population was statistically significant, its clinical relevance should be retested in larger trials. Moreover, despite observed changes in IOP in this study, the number of patients with glaucoma was not large enough to extend the conclusion to this population. Even though these factors limit the power of the current study, the results do present valuable information for future study design. In future, trials evaluating the role of glucosamine on IOP may represent more relevant population, with larger sample size and longer follow-up.

In conclusion, based on the results of this study, glucosamine causes statistically significant rise in IOP, which is more pronounced in elderly patients. Clinical implication of this finding in glaucoma patients needs further evaluation in larger studies with longer follow-up and in more relevant population.

\section{Summary}

What was known before
It was known that glycosaminoglycans have an important
role in the morphology and function of the trabecular
meshwork.
- Deposition and accumulation of glycosaminoglycans have
been shown in open angle and steroid-induced glaucoma,
which is responsible for high intraocular pressure in these
conditions.
What this study adds
Exogenous glucosamine, as the most commonly used
supplementary medication that is prescribed for
osteoarthritis, may lead to high intraocular pressure.
The importance of this finding should be assessed in
longer-term study and its relevance to glaucoma patients
mandate further evaluation.

\section{Conflict of interest}

The authors declare no conflict of interest. 


\section{References}

1 Deal CL, Moskowitz RW. Nutraceuticals as therapeutic agents in osteoarthritis. The role of glucosamine, chondroitin sulfate and collagen hydrolysate. Rheum Dis Clin North Am 1999; 25: 379-395.

2 Altman RD, Hochberg M, Moskowitz RW, Schnitzer J. On behalf of the American College of Rheumatology Subcommittee on Osteoarthritis Guidelines. Recommendations for the medical management of osteoarthritis on the hip and the knee. Arthritis Rheum 2000; 43: 1905-1915.

3 Bucsi L, Poór G. Efficacy and tolerability of oral chondroitin sulfate as a symptomatic slow-acting drug for osteoarthritis (SYSADOA) in the treatment of knee osteoarthritis. Osteoarthr Cartil 1998; 6: 31-36.

4 Richy F, Bruyere H, Ethgen O, Cucherat M, Henrotin Y, Reginster JY. Structural and symptomatic efficacy of glucosamine and chondroitin in knee osteoarthritis: a comprehensive meta-analysis. Arch Intern Med 2003; 163: 1514-1522.

5 Nagaoka I, Igarashi M, Sakamoto K. Biological activities of glucosamine and its related substances. Adv Food Nutr Res 2012; 65: 337-352.

6 Acott TS, Westcott M, Passo MS, Van Buskirk EM. Trabecular meshwork glycosaminoglycans in human and cynomolgus monkey eyes. Invest Ophthalmol Vis Sci 1985; 26: 1320-1329.

7 Knepper PA, McLone DG. Glycosaminoglycans and outflow pathways of the eye and brain. Pediatr Neurosci 1986; 12: 240-251.

8 Pavelka K, Gatterova J, Olejarova M, Machacek S, Giacovelli G, Rovati LC. Glucosamine sulfate use and delay of progression of knee osteoarthritis: a 3-year, randomized, placebocontrolled, double-blind study. Arch Intern Med 2002; 162: 2113-2123.

9 Reginster JY, Deroisy R, Rovati LC, Lee RL, Lejeune E, Bruyere $\mathrm{O}$ et al. Long-term effects of glucosamine sulphate on osteoarthritis progression: a randomised, placebocontrolled clinical trial. Lancet 2001; 357: 251-256.

10 Singh JA, Wilt T, MacDonald R. Chondroitin for osteoarthritis. Cochrane Database Syst Rev 2006; 1: CD005614.

11 Towheed TE, Maxwell L, Anastassiades TP, Shea B, Houpt J, Robinson $\mathrm{V}$ et al. Glucosamine therapy for treating osteoarthritis. Cochrane Database Syst Rev 2005; 2: CD002946.

12 Murphy RK, Ketzler L, Rice RD, Johnson SM, Doss MS, Jaccoma EH. Oral glucosamine supplements as a possible ocular hypertensive agent. JAMA Ophthalmol 2013; 131: 955-957.

13 Ametek ultraprecision technology. Ocular Response Analyzer's User Guide (Revision B). Buffalo, NY: Reichert Ophthalmic Instruments, 2005. Available at http://
doclibrary.com/MSC167/PRM/16070-101-Rev-BUG51371538.pdf.

14 McAlindon TE, LaValley MP, Gulin JP, Felson DT. Glucosamine and chondroitin for treatment of osteoarthritis: a systematic quality assessment and meta-analysis. JAMA 2000; 283: 1469-1475.

15 Sawitzke AD, Shi H, Finco MF, Dunlop DD, Harris CL, Singer NG et al. Clinical efficacy and safety of glucosamine, chondroitin sulphate, their combination, celecoxib or placebo taken to treat osteoarthritis of the knee: 2-year results from GAIT. Ann Rheum Dis 2010; 69: 1459-1464.

16 Johnson KA, Hulse DA, Hart RC, Kochevar D, Chu Q. Effects of an orally administered mixture of chondroitin sulfate, glucosamine hydrochloride and manganese ascorbate on synovial fluid chondroitin sulfate 3B3 and 7D4 epitope in a canine cruciate ligament transection model of osteoarthritis. Osteoarthritis Cartilage 2001; 9: 14-21.

17 Rohen JW, Schachtschabel DO, Wehrmann R. Structural changes of human and monkey trabecular meshwork following in vitro cultivation. Graefes Arch Clin Exp Ophthalmol 1982; 218: 225-232.

18 Rohen JW, Futa R, Lütjen-Drecoll E. The fine structure of the cribriform meshwork in normal and glaucomatous eyes as seen in tangential sections. Invest Ophthalmol Vis Sci 1981; 21: $574-585$.

19 Binninger EA, Schachtschabel DO, Rohen JW. Exogenous glycosaminoglycans stimulate hyaluronic acid synthesis by cultured human trabecular-meshwork cells. Exp Eye Res 1987; 45: 169-177.

20 Jones R 3rd, Rhee DJ. Corticosteroid-induced ocular hypertension and glaucoma: a brief review and update of the literature. Curr Opin Ophthalmol 2006; 17: 163-167.

21 Meek KM, Leonard DW. Ultrastructure of the corneal stroma: a comparative study. Biophys J 1993; 64: 273-280.

22 Laverty S, Sandy JD, Celeste C, Vachon P, Marier JF, Plaas AH. Synovial fluid levels and serum pharmacokinetics in a large animal model following treatment with oral glucosamine at clinically relevant doses. Arthritis Rheum 2005; 52: 181-191.

23 Becker B. The decline in aqueous secretion and out flow facility with age. Am J Ophthalmol 1958; 46: 731-736.

24 Gaasterland D, Kupfer C, Milton R, Ross K, McCain L, MacLellan H. Studies of aqueous humour dynamics in man. VI. Effect of age upon parameters of intraocular pressure in normal human eyes. Exp Eye Res 1978; 26: 651-656.

25 Vranka JA, Kelley MJ, Acott TS, Keller KE. Extracellular matrix in the trabecular meshwork: intraocular pressure regulation and dysregulation in glaucoma. Exp Eye Res 2015; 133: 112-125.

26 Weekers R, Watillon M, De Rudder M. Experimental and clinical investigations into the resistance to out flow of aqueous humour in normal subjects. Br J Ophthalmol 1956; 40: $225-233$. 